\title{
Sexual Arousal
}

National Cancer Institute

\section{Source}

National Cancer Institute. Sexual Arousal. NCI Thesaurus. Code C73522.

Heightened urges of sexual desires in preparation for sexual behavior. 